\title{
A avaliação do método tomográfico glenoid track na ressonância magnética/artro RM
}

\section{Evaluation of the Glenoid Track Tomographic Method in Magnetic Resonance Imaging/Arthro-MRI}

\author{
Andre Couto Godinho' ${ }^{10}$ Pedro Couto Godinho ${ }^{1}$ Flávio de Oliveira França2 ${ }^{20}$ \\ Elísio José Salgado Ribeiro ${ }^{3(0)}$ Daniel Carvalho de Toledo ${ }^{2(0)}$ Guilherme Henrique Franco ${ }^{20}$
}

${ }^{1}$ Hospital Ortopédico, Belo Horizonte, MG, Brasil

2 Ortopédico BH, Belo Horizonte, MG, Brasil

${ }^{3}$ Axial Medicina Diagnóstica, Belo Horizonte, MG, Brasil
Endereço para correspondência Daniel Carvalho de Toledo, MD, Ortopédico BH, Belo Horizonte, Minas Gerais, Brasil (e-mail: danieltoledo@me.com).

Rev Bras Ortop 2021;56(6):733-740.

\section{Resumo}

\section{Palavras-chave}

- cavidade glenoide

- instabilidade anterior do ombro

- luxação do ombro
Objetivo Comparar a avaliação do método glenoid-track (GT) em exames de tomografia computadorizada com reconstrução 3-D (TC-3D) com a avaliação realizada em exames de ressonância magnética (RM) e/ou artro-ressonância magnética (ARM). Métodos Quarenta e quatro ombros com diagnóstico clínico e radiográfico de instabilidade anterior traumática foram avaliados por meio de exames de TC-3D, RM e/ou ARM. As variáveis GT, intervalo de Hill-Sachs (IHS) e a perda óssea da glenoide (POG) foram realizadas por um médico radiologista, utilizando imagens de TC-3D, e classificadas em on-track/off-track. Três cirurgiões cegos à avaliação do radiologista realizaram o mesmo método utilizando RM/ARM. O estudo realizou análise descritiva, de variância, de associação da discordância de resultados, de concordância e curva característica de operação do receptor.

Resultados Os resultados dos 4 examinadores foram totalmente concordantes em $61,4 \%$. A RM/ARM diagnosticou lesões off-track com a sensibilidade variando de 35 a $65 \%$, e lesões on-track com a especificidade variando de 91,67 a $95,83 \%$. A acurácia variou de 68,1 a $79,5 \%$. A maior divergência de dados ocorreu para o diagnóstico por RM/ARM de lesões off-track. A maior variabilidade dos dados ocorreu para o cálculo do IHS. Valores maiores de IHS e de POG foram associados a maior discordância entre os examinadores. A RM/ARM apresentou menor medida de valores de IHS quando comparado com a TC-3D. Ocorreu apenas moderada concordância no método GT entre a TC e a RM/ARM (Kappa 0,325-0,579).

Conclusão A RM/ARM apresentou baixa acurácia e moderada concordância para o método GT, devendo ser utilizada com cautela por cirurgiões. recebido

09 de Dezembro de 2019

aceito

06 de Julho de 2020

Publicado on-line

Outubro 29, 2020
DOI https://doi.org/

$10.1055 / \mathrm{s}-0040-1716766$ ISSN 0102-3616. (c) 2020. Sociedade Brasileira de Ortopedia e Traumatologia. All rights reserved.

This is an open access article published by Thieme under the terms of the Creative Commons Attribution-NonDerivative-NonCommercial-License, permitting copying and reproduction so long as the original work is given appropriate credit. Contents may not be used for commercial purposes, or adapted, remixed, transformed or built upon. (https://creativecommons.org/ licenses/by-nc-nd/4.0/)

Thieme Revinter Publicações Ltda., Rua do Matoso 170, Rio de Janeiro, RJ, CEP 20270-135, Brazil 


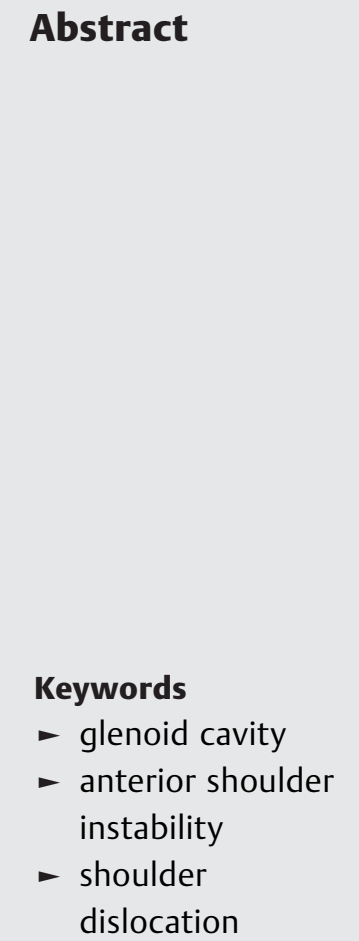

Objective To evaluate and compare the glenoid track method in 3D-reconstructed computed tomography (3D-CT) scans with magnetic resonance imaging (MRI) and/or arthro-MRI.

Methods Forty-four shoulders with clinical and radiographic diagnosis of traumatic anterior instability were assessed using 3D-CT, MRI, and/or arthro-MRI scans. Glenoid track (GT), Hill-Sachs interval (HSI), and glenoid bone loss (GBL) were determined by a radiologist using 3D-CT images, and classified as on-track/off-track. Three surgeons, blinded to the radiologist's evaluation, performed the same determinations using MRI/arthro-MRI. Descriptive analysis, variance analysis, results disagreement analysis, and receiver operating characteristic (ROC) curves were performed.

Results Results from the 4 examiners were fully consistent in $61.4 \%$ of the cases. MRI/arthro-MRI diagnosed off-track injuries with 35 to $65 \%$ sensitivity and on-track injuries, with 91.67 to $95.83 \%$ specificity. Accuracy ranged from 68.1 to $79.5 \%$. The greatest data divergence occurred for off-track injuries diagnosed by MRI/arthro-MRI. The greatest data variability referred to HSI calculation. Higher HSI and GBL values were associated with greater disagreement among examiners. Hill-Sachs interval values were lower at MRI/arthro-MRI when compared to 3D-CT. Agreement between CT and MRI/arthro-MRI for the GT method was only moderate (kappa value, $0.325-0.579$ ).

Conclusion Magnetic resonance imaging/arthro-MRI showed low accuracy and moderate agreement for the GT method; as such, it should be used with caution by surgeons.

\section{Introdução}

A avaliação adequada do paciente com instabilidade anterior traumática do ombro permanece um desafio para os ortopedistas. ${ }^{1}$ Para o estudo preciso e detalhado das lesões de partes moles e ósseas na instabilidade glenoumeral, exames de tomografia computadorizada com reconstrução 3-D (TC3D), ressonância magnética (RM) ou artro-ressonância magnética (ARM) são recomendados, o que pode aumentar os custos da propedêutica, retardar o tratamento e burocratizar a conduta por parte do médico assistente. ${ }^{2}$

A observação da interação dinâmica entre as perdas ósseas do úmero proximal e da glenoide na luxação anterior traumática do ombro - bipolaridade das lesões foi descrita na TC-3D através do conceito glenoid track (GT). $\mathrm{O}$ mesmo tem ganhado destaque no meio científico pela sua capacidade prognóstica e por orientar o tratamento adequado de acordo com a classificação da lesão de HillSachs (LHS) e o tamanho da perda óssea da glenoide (POG). ${ }^{3}$

Considerando esse método como ideal e buscando diminuir custos com a avaliação simultânea das lesões de partes moles e ósseas em um único exame, a literatura ortopédica tem investido esforços para substituir a TC-3D pela RM/ARM..$^{3-5}$ Nesse sentido, o objetivo do presente estudo é comparar a avaliação do método GT realizada por cirurgiões de ombro utilizando RM/ARM, com a avaliação realizada por um médico radiologista utilizando TC-3D. Como hipótese, acreditamos que a aferição do GT na RM/ARM pode ser realizada de forma acurada e confiável por cirurgiões em sua prática clínica.

\section{Material}

Trata-se de um estudo transversal, analítico de 43 pacientes, 44 ombros, selecionados em método não probabilístico amostragem por conveniência - com diagnóstico de instabilidade anterior traumática do ombro, atendidos no período de março de 2015 a setembro de 2018. Todos os pacientes foram submetidos a exames de TC-3D, RM e/ou ARM em uma única clínica de radiologia.

O cálculo do GT, IHS, da POG e a classificação das lesões em on-track/off-track foi realizada em todos exames de TC-3D por apenas um médico radiologista $(R)$, com mais de 10 anos de experiência na área musculoesquelética. Esta análise foi considerada o padrão de referência.

Três cirurgiões especialistas em ombro (C1, C2 e C3), capacitados para o método GT, realizaram os mesmos cálculos em exames de RM ou ARM. Todos foram cegados para os resultados obtidos por $\mathrm{R}$ e para os resultados dos demais avaliadores. Todos os cálculos foram realizados em um só momento e registrados por dois médicos residentes especialistas em cirurgia do ombro (R4).

Não foram consideradas as variáveis gênero, idade e dominância. Foram incluídos pacientes com diagnóstico clínico e radiológico de instabilidade anterior traumática do ombro, com documentação completa de exames de TC-3D, RM e/ou ARM e que não realizaram tratamento cirúrgico até o momento da análise dos dados. Pacientes com exames realizados em outras clínicas radiológicas, avaliados por outro radiologista, com informações incompletas e lesões associadas como fraturas, lesões do manguito rotador ou artrose glenoumeral foram excluídos. 

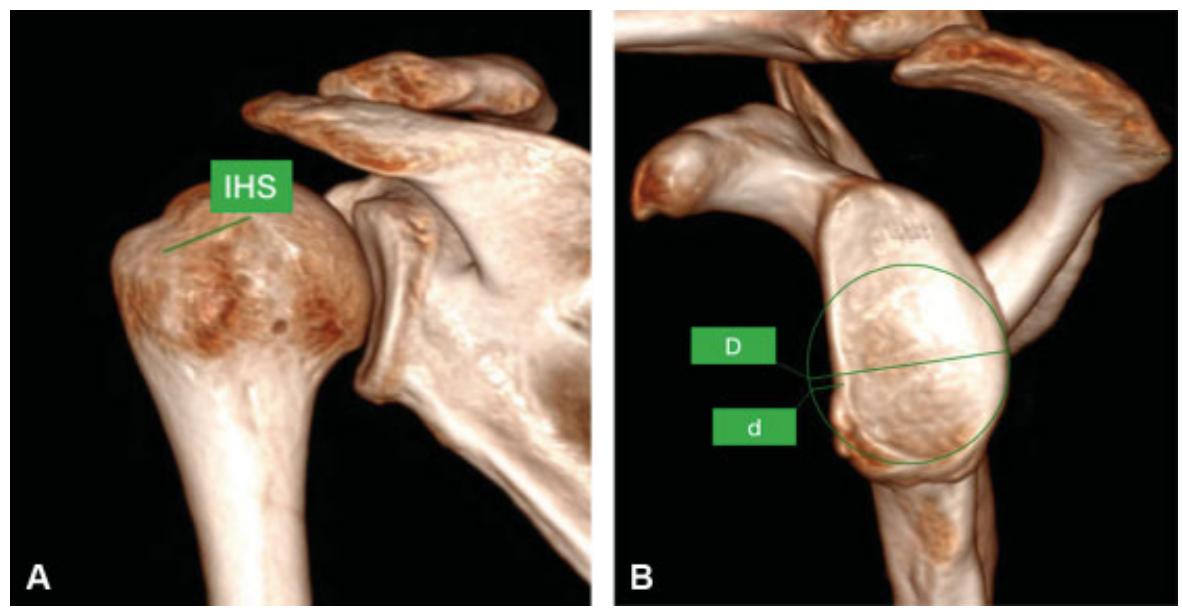

Fig. 1 Imagens tomográficas computadorizadas tridimensionais demonstrando o cálculo do glenoid track (GT). (1A) O intervalo do Hill-Sachs (IHS) corresponde à distância entre a margem interna do footprint do manguito rotador e a borda medial do defeito ósseo. (1B) A partir de um círculo virtual traçado nos dois terços inferiores da glenoide, tendo como referência suas bordas íntegras, obtemos a medida de qual seria o diâmetro da mesma, na ausência de defeito ósseo (D) e da largura do defeito ósseo (d). O GT corresponde 83\% do diâmetro glenoidal menos o valor do defeito ósseo.

\section{Métodos}

Todos os pacientes realizaram a TC-3D em um scanner Siemens Somatom Sensation de 64 canais (Siemens AG, Munique, Alemanha) ou Siemens Somatom Definition AS de 128 canais (Siemens AG), em posição supina e com o membro avaliado em rotação neutra.

Os exames de RM e ARM (Magnetom Essenza 1,5 T - Siemens Healthcare, Erlangen, Alemanha) foram realizados, em decúbito dorsal, com o ombro em rotação neutra, sendo usadas sequências ponderadas em $\mathrm{T} 2$ sem ou com supressão de gordura (TR/TE $2280 / 42$, FOV $160 \times 100 \mathrm{~mm}$, matriz $384 \times 70$, espessura de $3 \mathrm{~mm}$ ), no plano axial, sagital e coronal pós-processadas (Kodak Carestream PACS, Belo Horizonte, Minas Gerais, Brasil).

O cálculo das variáveis GT, do IHS, da POG e a classificação on-track/off-track foi realizada em exames de TC-3D conforme

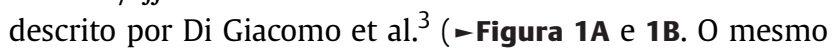
cálculo foi realizado em exames de RM/ARM seguindo a técnica descrita por Guyftpoulos et al. ${ }^{4}$ (-Figura 2A e 2B). O GT foi calculado utilizando a fórmula $\mathrm{GT}=0,83 \mathrm{D}-\mathrm{d}$.

A análise dos dados foi realizada no programa IBM SPSS versão 23.0. (IBM Corp., Armonk, NY, EUA). O nível de significância utilizado em todo estudo foi de 5\%. Este estudo foi aprovado pelo Comitê de Ética em Pesquisa da instituição.

\section{Resultado}

\section{Análise descritiva}

Nenhum dos 44 ombros da amostra foi excluído do estudo. Todos foram submetidos a exames de TC-3D, com 19 (43,2\%) submetidos adicionalmente a exames de RM, e 25 (56,8\%) exames de ARM. Vinte e quatro ombros $(65,9 \%)$ foram classificados como on-track e $20(45,5 \%)$ como off-track pelo examinador $\mathrm{R}$ em exames de TC-3D. 0 resultado dos 4 avaliadores foi totalmente concordante em $61,4 \%$ dos casos (-Tabela 1).
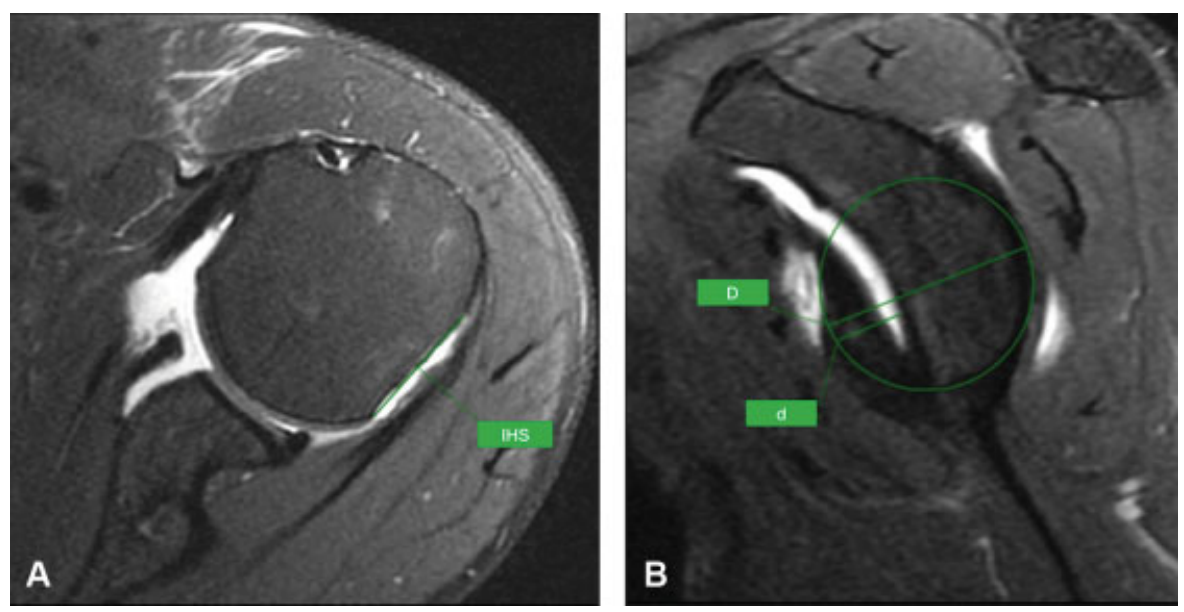

Fig. 2 Imagens de artro-ressonância demonstrando a medida do glenoid-track (GT). Para o cálculo do GT, o diâmetro (D) da glenoide inferior foi obtido por meio de um círculo desenhado ao longo da glenoide inferior. O comprimento da perda óssea da glenoide (d) foi então aferido. $\mathrm{O}$ glenoid-track foi calculado utilizando a fórmula GT =0,83D - d. (2A) Medida do intervalo de Hill-Sachs utilizando uma vista posterior da cabeça umeral. (2B) Cálculo do GT semelhante ao realizado nas imagens de tomografia computadorizada. 
Tabela 1 Análise descritiva das variáveis qualitativas: exame de imagem realizado, classificação on-track/off-track, e discordância entre os examinadores

\begin{tabular}{|l|l|l|}
\hline Variável & Frequência & $\%$ \\
\hline Exame de imagem realizado & & \\
\hline TC & 44 & 100,0 \\
\hline RM & 19 & 43,2 \\
\hline ARM & 25 & 56,8 \\
\hline Resultado - R & & \\
\hline On-track & 24 & 54,5 \\
\hline Off-track & 20 & 45,5 \\
\hline Resultado - C1 & & \\
\hline On-track & 29 & 65,9 \\
\hline Off-track & 15 & 34,1 \\
\hline Resultado - C2 & & \\
\hline On-track & 31 & 70,5 \\
\hline Off-track & 13 & 29,5 \\
\hline Resultado - C3 & & \\
\hline On-track & 36 & 81,8 \\
\hline Off-track & 8 & 18,2 \\
\hline Discordância & & \\
\hline 0 & 27 & 61,4 \\
\hline 1 & 7 & 15,9 \\
\hline 2 & 5 & 11,4 \\
\hline 3 & 5 & 11,4 \\
\hline Total & 44 & 100,0 \\
\hline
\end{tabular}

Abreviaturas: ARM, artro-ressonância magnética; RM, ressonância magnética; TC, tomografia computadorizada.

\section{Análise de variância}

Para avaliar a variação dos resultados do GT, IHS e POG em relação aos quatro examinadores, foram aplicados os testes de Kolmogorov-Smirnov, de Levene, Posthoc de Dunnet e o Dunnet T3.

Foi comprovado diferença estatística significativa nos valores de GT, IHS e POG quando se compararam os quatro examinadores. A maior variabilidade de dados ocorreu para a variável IHS. A média dos valores do IHS na RM/ARM foram menores quando comparado com a TC-3D (-Tabelas 2 e 3 ).

O Posthoc para a variável GT teve como resultado uma diferença estatisticamente significativa entre os examinadores $\mathrm{R}$ e $\mathrm{C} 3(P=0,027)$ e entre os examinadores $\mathrm{C} 1$ e C3 $(P=0,039)$, sendo que os valores encontrados por $\mathrm{C} 3$ foram significativamente maiores que os outros dois.

Para a variável IHS o resultado foi de diferença significativa entre os examinadores $\mathrm{R}$ e $\mathrm{C} 2(P=0,017)$ e entre os examinadores $\mathrm{R}$ e C3 $(P=0,001)$, sendo que os valores encontrados por $\mathrm{R}$ foram significativamente maiores.

Já para a POG foi encontrada diferença entre os examinadores C1 e C3 $(P=0,027)$, onde os valores encontrados por C1 foram significativamente maiores em comparação aos valores obtidos por C3.

\section{Análise de associação da discordância de resultados}

Para a avaliação das variáveis quantitativas, foi realizada uma análise de correlação não paramétrica. Para isso os resultados de $\mathrm{R}$ foram sempre considerados corretos e o número de discordâncias desse resultado foi avaliado (0-3 discordâncias). O teste utilizado foi a correlação de Spearman.

Houve correlação estatisticamente significativa entre IHS e a POG, ambas com coeficiente positivo, indicando que

Tabela 2 Análise descritiva das variáveis quantitativas: glenoid track, intervalo de Hill-Sachs e perda óssea da glenoide conforme os examinadores $\mathrm{R}, \mathrm{C} 1, \mathrm{C} 2$ e $\mathrm{C} 3$

\begin{tabular}{|c|c|c|c|c|c|c|c|}
\hline & n & Média & Mediana & Desvio padrão & Mínimo & Máximo & Amplitude interquartil \\
\hline $\begin{array}{l}\text { Examinador } \mathrm{R} \\
\text { GT }\end{array}$ & 44 & 20,30 & 20,27 & 2,50 & 12,98 & 27,39 & 3,13 \\
\hline IHS & 44 & 19,64 & 20,21 & 3,61 & 11,32 & 29,01 & 4,17 \\
\hline POG & 44 & 8,68 & 9,50 & 7,65 & 0,00 & 33,00 & 14,00 \\
\hline $\begin{array}{l}\text { Examinador } \mathrm{C} 1 \\
\text { GT }\end{array}$ & 44 & 20,00 & 19,98 & 3,71 & 2,46 & 26,08 & 3,18 \\
\hline IHS & 44 & 17,36 & 18,35 & 5,72 & 6,35 & 33,84 & 8,16 \\
\hline POG & 44 & 11,63 & 11,50 & 7,12 & 0,00 & 33,00 & 7,58 \\
\hline $\begin{array}{l}\text { Examinador C2 } \\
\text { GT }\end{array}$ & 44 & 20,60 & 20,68 & 2,73 & 12,08 & 25,04 & 4,00 \\
\hline IHS & 44 & 16,62 & 16,10 & 5,39 & 3,78 & 29,82 & 5,70 \\
\hline POG & 44 & 7,83 & 0,00 & 9,19 & 0,00 & 30,53 & 15,43 \\
\hline $\begin{array}{l}\text { Examinador C3 } \\
\text { GT }\end{array}$ & 44 & 21,95 & 22,13 & 2,81 & 15,49 & 30,52 & 3,55 \\
\hline IHS & 44 & 15,69 & 14,51 & 5,30 & 4,79 & 29,35 & 6,46 \\
\hline POG & 44 & 7,21 & 7,00 & 7,25 & 0,00 & 22,00 & 13,00 \\
\hline
\end{tabular}

Abreviaturas: GT, glenoid track; IHS, intervalo de Hill-Sachs; POG, perda óssea da glenoide. 
Tabela 3 Medidas descritivas das variáveis glenoid track, intervalo de Hill-Sachs e perda óssea da glenoide para análise de variância conforme os examinadores R, C1, C2 e C3

\begin{tabular}{|c|c|c|c|c|c|c|c|c|c|}
\hline & & \multirow[t]{2}{*}{$n$} & \multirow[t]{2}{*}{ Média } & \multirow[t]{2}{*}{ Desvio padrão } & \multirow[t]{2}{*}{ Erro padrão } & \multicolumn{2}{|c|}{$\begin{array}{l}\text { Intervalo de confiança de } 95 \% \\
\text { para média }\end{array}$} & \multirow[t]{2}{*}{ Mínimo } & \multirow[t]{2}{*}{ Máximo } \\
\hline & & & & & & Limite inferior & Limite superior & & \\
\hline \multirow[t]{5}{*}{ GT } & $\mathrm{R}$ & 44 & 20,30 & 2,50 & 0,38 & 19,54 & 21,06 & 12,98 & 27,39 \\
\hline & $\mathrm{C} 1$ & 44 & 20,00 & 3,71 & 0,56 & 18,87 & 21,13 & 2,46 & 26,08 \\
\hline & $\mathrm{C} 2$ & 44 & 20,60 & 2,73 & 0,41 & 19,76 & 21,43 & 12,08 & 25,04 \\
\hline & $\mathrm{C3}$ & 44 & 21,95 & 2,81 & 0,42 & 21,10 & 22,81 & 15,49 & 30,52 \\
\hline & Total & 176 & 20,71 & 3,04 & 0,23 & 20,26 & 21,17 & 2,46 & 30,52 \\
\hline \multirow[t]{5}{*}{ IHS } & $\mathrm{R}$ & 44 & 19,64 & 3,61 & 0,54 & 18,55 & 20,74 & 11,32 & 29,01 \\
\hline & C1 & 44 & 17,36 & 5,72 & 0,86 & 15,62 & 19,10 & 6,35 & 33,84 \\
\hline & $\mathrm{C} 2$ & 44 & 16,62 & 5,39 & 0,81 & 14,98 & 18,26 & 3,78 & 29,82 \\
\hline & $\mathrm{C3}$ & 44 & 15,69 & 5,30 & 0,80 & 14,08 & 17,30 & 4,79 & 29,35 \\
\hline & Total & 176 & 17,33 & 5,24 & 0,39 & 16,55 & 18,11 & 3,78 & 33,84 \\
\hline \multirow[t]{5}{*}{ POG } & $\mathrm{R}$ & 44 & 8,68 & 7,65 & 1,15 & 6,36 & 11,01 & 0,00 & 33,00 \\
\hline & C1 & 44 & 11,63 & 7,12 & 1,07 & 9,46 & 13,79 & 0,00 & 33,00 \\
\hline & $\mathrm{C} 2$ & 44 & 7,83 & 9,19 & 1,39 & 5,03 & 10,62 & 0,00 & 30,53 \\
\hline & $\mathrm{C} 3$ & 44 & 7,21 & 7,25 & 1,09 & 5,01 & 9,41 & 0,00 & 22,00 \\
\hline & Total & 176 & 8,84 & 7,96 & 0,60 & 7,65 & 10,02 & 0,00 & 33,00 \\
\hline
\end{tabular}

Abreviaturas: GT, glenoid track; IHS, intervalo de Hill-Sachs; POG, perda óssea da glenoide.

Tabela 4 Análise de correlação das variáveis glenoid track (GT), intervalo de Hill-Sachs (IHS), perda óssea da glenoide (POG) e discordância entre os examinadores

\begin{tabular}{|c|c|c|c|c|c|}
\hline \multicolumn{6}{|l|}{ Correlações } \\
\hline & & & IHS & POG & Discordância \\
\hline \multirow[t]{9}{*}{$\rho$ de Spearman } & \multirow[t]{3}{*}{ GT } & Coeficiente de correlação & 0,154 & $-0,565$ & $-0,264$ \\
\hline & & $P$ & 0,320 & 0,000 & 0,084 \\
\hline & & $n$ & 44 & 44 & 44 \\
\hline & \multirow[t]{3}{*}{ IHS } & Coeficiente de correlação & 1,000 & 0,177 & 0,434 \\
\hline & & $P$ & & 0,249 & 0,003 \\
\hline & & $n$ & 44 & 44 & 44 \\
\hline & \multirow[t]{3}{*}{ POG } & Coeficiente de correlação & & & 0,334 \\
\hline & & $P$ & & & 0,027 \\
\hline & & $\mathrm{n}$ & & & 44 \\
\hline
\end{tabular}

Abreviaturas: GT, glenoid track; IHS, intervalo de Hill-Sachs; POG, perda óssea da glenoide.

valores maiores de IHS e POG foram associados a mais discordâncias entre os examinadores ( - Tabela 4 ).

Para a avaliação das variáveis qualitativas utilizou-se o teste Qui-quadrado de Pearson. Observa-se que $88,24 \%$ das discordâncias foram para as lesões off-track. Isso demonstra que a maior dificuldade ocorreu no diagnóstico por RM/ARM das lesões do tipo off-track calculadas por TC-3D.

\section{Análise de concordância}

Observa-se que os valores de sensibilidade não foram muito altos variando de 35 a $65 \%$ (-Tabela 5). Em todos os casos o coeficiente Kappa foi estatisticamente significativo. O coefici- ente Kappa é utilizado para descrever a concordância entre dois ou mais testes, variando de 0 a 1 . Valores próximos de 0 indicam baixa concordância e valores próximos a 1 , alta concordância. 0 índice de Kappa variou de 0,325 a 0,579. Isso significa que o método GT utilizando imagens de RM/ARM apresentou apenas moderada capacidade de identificar pacientes com lesão off-track diagnosticadas por imagens de TC-3D.

O valor preditivo positivo (VPP) variou de 86,67 a $92,31 \%$. O valor preditivo negativo (VPN), variou de 63,89 a $75,86 \%$. A acurácia diagnóstica para lesões off-track variou de 68,1 a $79,5 \%$ entre os examinadores. 
Tabela 5 Índice Kappa, sensibilidade, especificidade, valor preditivo, razão de verossimilhança e acurácia dos examinadores cirurgiões (C1, C2 e C3) no diagnóstico por RM/ARM de lesões ontrack/off-track

\begin{tabular}{|l|l|l|l|}
\hline & C1 & C2 & C3 \\
\hline Índice Kappa & 0,579 & 0,575 & 0,325 \\
\hline Sensibilidade (\%) & 65,00 & 60,00 & 35,00 \\
\hline Especificidade (\%) & 91,67 & 95,83 & 95,83 \\
\hline Valor preditivo positivo (\%) & 86,67 & 92,31 & 87,50 \\
\hline Valor preditivo negativo (\%) & 75,86 & 74,19 & 63,89 \\
\hline $\begin{array}{l}\text { Razão de verossimilhança } \\
\text { positiva }\end{array}$ & 7,80 & 14,39 & 8,39 \\
\hline $\begin{array}{l}\text { Razão de verossimilhança } \\
\text { negativa }\end{array}$ & 0,38 & 0,42 & 0,68 \\
\hline Acurácia & 79,5 & 79,5 & 68,1 \\
\hline Valor de $p$ & $<0,001$ & $<0,001$ & 0,014 \\
\hline
\end{tabular}

Abreviaturas: ARM, artro-ressonância magnética; RM, ressonância magnética.

\section{Curva COR}

Observa-se pelos resultados da curva característica de operação do receptor (COR) que apenas a avaliação de C3 não foi estatisticamente eficiente no diagnóstico de lesões off-track $(P>0,05)$ (-Figura 3). O melhor resultado foi o da classificação realizada por $\mathrm{C} 1$, o qual obteve a maior área sob a curva. Quanto melhor o teste, mais a área sob a curva COR se aproxima de 1 .

\section{Discussão}

Historicamente os estudos sobre instabilidade anterior do ombro focaram na POG, ocorrida durante o episódio de luxação do ombro, também chamada lesão de Bankart ósseo (LBO). ${ }^{6,7}$ É amplamente aceito que este dano estrutural altera a biomecânica da glenoide ao prejudicar sua função de estabilizador estático. ${ }^{8}$ Defeitos maiores que 20 a $25 \%$ merecem especial atenção por apresentarem pior prognóstico com o reparo artroscópico. 6,9

Quando o ombro sofre luxação anterior uma fratura por impacção pode ocorrer na porção póstero-superior e lateral da cabeça umeral. Esta lesão é definida como lesão de HillSachs e está presente em até 70\% das luxações primárias do ombro. ${ }^{10,11}$ Estudos revelam que defeitos ósseos com 5/8 do raio da cabeça umeral já seriam capazes de alterar a instabilidade do ombro. ${ }^{12}$

Embora já demonstrado a importância da LHS e da LBO na biomecânica do ombro, a descrição do método GT permitiu a compreensão da interação dinâmica entre estas lesões ósseas. ${ }^{13}$ Atualmente, o método GT faz parte da rotina da maioria dos serviços de ortopedia, por sua capacidade prognóstica na avaliação das LHS e por ser capaz de orientar o tratamento da instabilidade anterior do ombro. ${ }^{3}$
A mensuração do GT e POG foi originalmente descrita para exames de TC-3D com o intuito de prever lesões do tipo engage. $^{3,13}$ Contudo, em busca de reduzir os custos, bem como a exposição à radiação, pesquisadores tem estudado o uso da RM/ARM para este objetivo. ${ }^{1,4,14}$

$O$ conceito de engagement foi inicialmente proposto por Burkhart e De Beer ${ }^{7}$ para explicar fatores relacionados a recidiva da instabilidade após a cirurgia de Bankart artroscópica. Pacientes com estas lesões, com o ombro abduzido e em rotação lateral estariam predispostos ao "encaixe" da LHS na borda anterior da glenoide levando à instabilidade articular. Lesões engage correspondem às lesões off-track no método GT. ${ }^{3}$

Gyftopoulos et al., ${ }^{4}$ com a intenção de viabilizar o método glenoid-track para a RM, classificaram o GT em 75 ombros e compararam o resultado com achados descritos de engagement durante a artroscopia. Concluíram que a RM apresenta sensibilidade de 72,2\% no diagnóstico de lesões engage (offtrack), especificidade de $87,9 \%$ para lesões não engage (ontrack), e acurácia geral de 84,2\%. O VPP foi de $65 \%$ e o VPN de $91,1 \%$

No presente estudo a RM/ARM diagnosticou lesões offtrack com a sensibilidade variando de 35 a $65 \%$ entre os cirurgiões, e lesões on-track com a especificidade variando de 91,67 a 95,83\%. A acurácia para o diagnóstico de lesões offtrack variou de 68,1 a 79,5\%. O VPP apresentou ao menos $86,67 \%$ de chance de identificar pacientes verdadeiramente off-track, enquanto o VPN apresentou ao menos 63,89\% de chance de identificar pacientes verdadeiramente positivos para lesões on-track. Em todos os casos o coeficiente Kappa foi significativo. Estes resultados são inferiores a aqueles demonstrados por Gyftopoulos et al. ${ }^{4}$

Schneider et al. ${ }^{15}$ avaliaram a variação da medida do GT intra e interobservadores em 71 pacientes com exames de TC-3D. Demonstraram que a avaliação da POG apresenta boa concordância intra e interobservadores ( $94 \%$ e $96 \%$, respectivamente). Contudo, um nível de confiabilidade de apenas $72 \%$ foi conseguido entre os examinadores na classificação on-track e off-track, considerado baixo pelos autores. Concluíram que a POG apresenta melhores níveis de reprodutibilidade e confiabilidade que a avaliação da LHS.

Nossos resultados demonstraram que a maior variabilidade dos dados ocorreu para o cálculo do IHS nos exames de RM/ARM, o mesmo demonstrado pelo estudo de Schnieder et al. ${ }^{15}$ em exames de TC-3D. Além disso, concluímos que a RM/ARM tende a apresentar menores valores de IHS quando comparado com a TC-3D. Várias etapas são necessárias para a avaliação adequada da LHS, em nossa opinião, e cada uma apresenta potencial de erro, o que leva à baixa confiabilidade e reprodutibilidade que a literatura tem demonstrado.

Funakoshi et al. ${ }^{16}$ avaliaram a concordância da classificação do GT entre exames de TC-3D e medidas realizadas intraoperatório em artroscopias. Dos 16 ombros classificados em on ou off-track, apenas 10 apresentaram concordância entre os 2 métodos (63\%, Kappa $=0,16$ ). Todos os casos que discordaram foram calculados como on-track pela TC-3D e off-track pela atroscopia. Concluíram que valores maiores de GT são medidos na TC-3D, quando comparados 


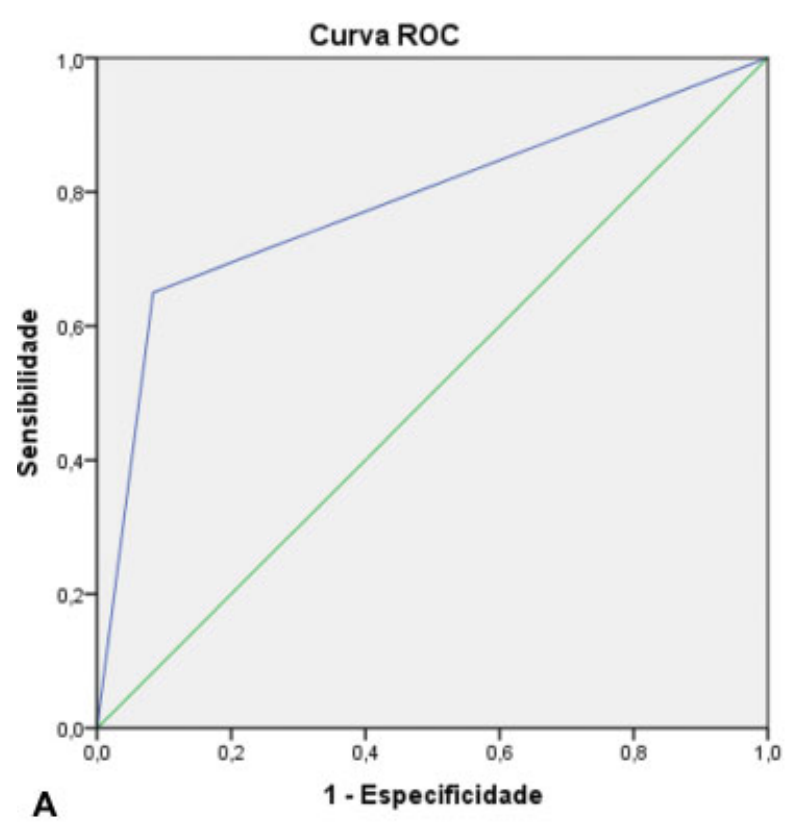

Os segmentos diagonais são produzidos por vinculos.

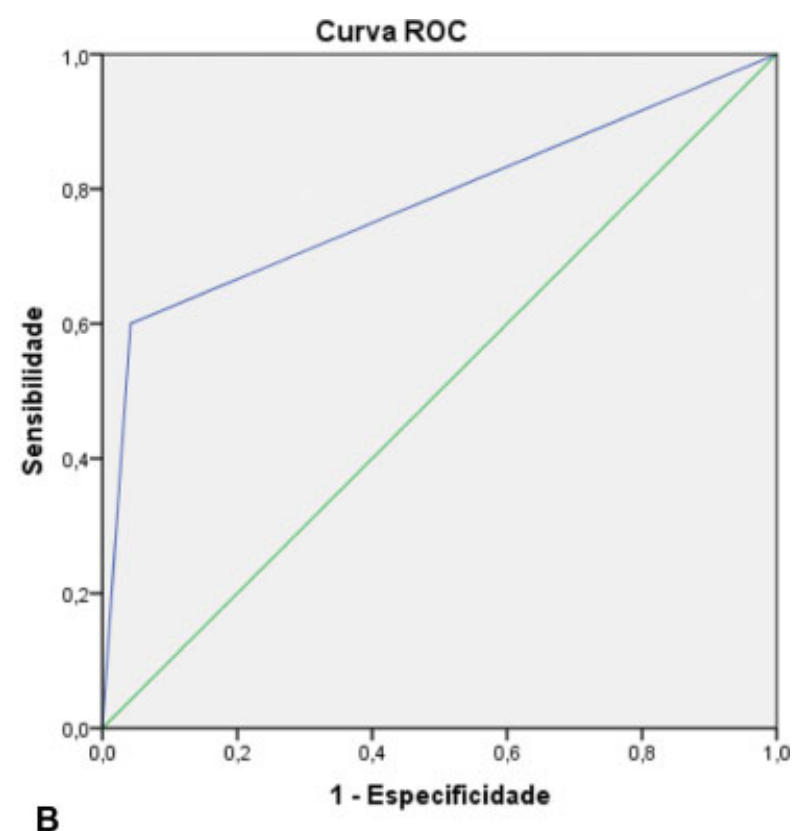

Os segmentos diagonais sắo produzidos por vínculos.

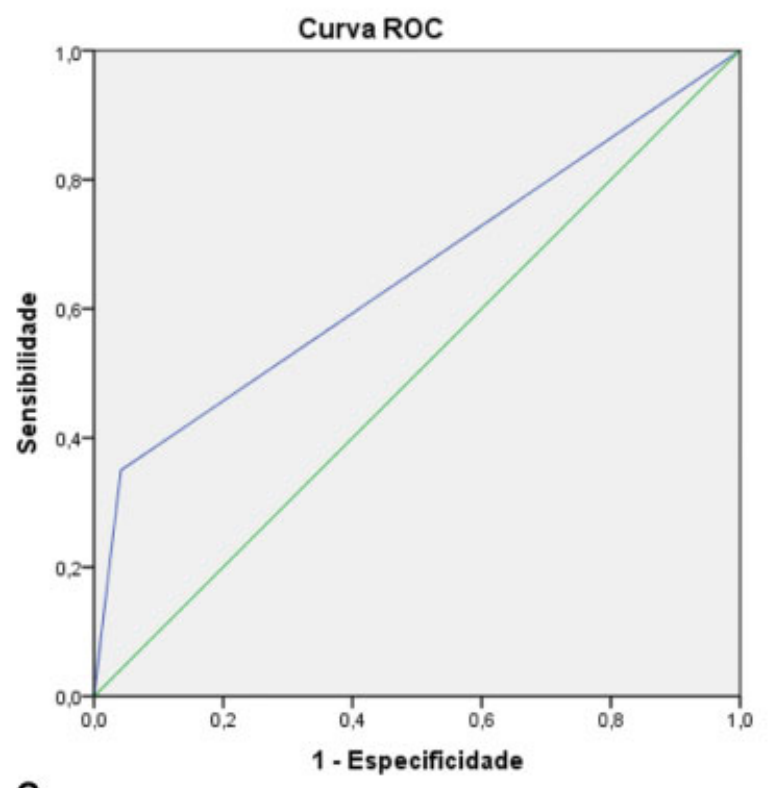

C

Os segmentos diagonais sâo produzidos por vinculos.

Fig. 3 Curvas características de operação do receptor (COR) dos examinadores cirurgiões (C1, C2 e C3) no diagnóstico por RM/ARM de lesões on-track/off-track. O resultado do cálculo da área sob a curva COR para os três examinadores foi: área $=0,783, P=0,001$ para o examinador C1; área $=0,779, P=0,002$ para o examinador $C 2$ e área $=0,654, P=0,081$ para o examinador $C 3$.

com a artroscopia, sendo responsáveis por toda discrepância e pela baixa concordância entre os métodos encontrada no estudo.

Nossos resultados revelaram moderada concordância na classificação on-track/off-track entre exames de TC-3D e RM/ARM (Kappa 0,325-0,579). Em nosso estudo a maioria dos resultados discordantes (15 de 17 resultados, ou 88,24\%) foram classificados como off-track pela TC-3D e on-track pela RM/ARM, o que poderia repercutir com uma abordagem inadequada da LHS, aumentando o risco de recidiva da instabilidade. Os resultados dos 4 examinadores foram totalmente concordantes em 27 dos 44 ombros $(61,4 \%)$ para a classificação on-track/off-track. Fato interessante é que valores maiores de IHS e de POG foram associados a maior discrepância entre os examinadores. Acreditamos que este fato pode ser explicado pela dificuldade dos avaliadores em selecionar as imagens mais representativas das referidas lesões ósseas, especialmente na RM/ARM que possibilita análise da imagem em apenas duas dimensões espaciais ao contrário da TC. 
Ao avaliar os resultados do cirurgião 3, observamos que este apresentou a maior média de valores de GT e foi o único a não apresentar eficiência estatística com a curva COR. Talvez por isso tenha apresentado a menor concordância dentre os examinadores (Kappa $=0,325$ ).

De nosso conhecimento, o presente estudo é o primeiro a comparar o método GT entre medidas na TC-3D com as medidas na RM/ARM. Apesar dos estudos disponíveis usarem diferentes modalidades para a comparação do método GT, nossos resultados se aproximam muito daqueles publicados na literatura.

Evidentemente, a utilização da RM/ARM na classificação do GT não é uma técnica perfeita. Como evidenciado nos resultados, a RM/ARM tende a apresentar menores valores de IHS. Nesse caso, acreditamos que a avaliação realizada pela TC-3D superestima os resultados por ser incapaz de identificar o ponto exato de inserção do tendão infraespinal, utilizado como referência para mensuração. Esta pode ser a explicação para a dificuldade da RM/ARM em identificar lesões off-track diagnosticadas pela TC-3D em nosso estudo. Este dado tem grande relevância pois, conforme descrito previamente, essas lesões devem ser abordadas pela técnica de remplissage para reduzir as chances de recidiva da luxação anterior do ombro. ${ }^{3}$

As razões da falha diagnóstica na classificação do GT já foram discutidas na literatura e podem estar relacionadas a variações inter e intraobservador, falha em determinar o ponto de inserção do manguito rotador no cálculo do IHS, falha em determinar a borda medial da LHS e falha em determinar o limite da POG. A estimativa do GT (componente mais importante da técnica) já foi citada como tecnicamente difícil e desafiadora. ${ }^{4,5}$

As limitações deste estudo incluem sua natureza transversal, assim como a amostragem limitada, realizada por método não probabilístico, atribuída principalmente ao custo de se realizar exames de tomografia computadorizada (TC) e RM/ARM. A avaliação realizada por cirurgiões, e não apenas radiologistas, e a ausência de avaliações seriadas das mesmas imagens constituem viés de aferição.

\section{Conclusão}

O método GT realizado em RM/ARM revelou baixa acurácia e moderada concordância no diagnóstico de lesões on-track/ off-track, quando comparado com a TC-3D. No que diz respeito ao cálculo do IHS, a definição da borda medial do tendão infraespinal e a borda medial da LHS deve ser instituída com atenção, uma vez que as maiores divergências dos dados ocorreram para esta medida. A RM/ARM tende a apresentar menores valores de IHS. A maior dificuldade do estudo envolveu o diagnóstico por RM/ARM de lesões offtrack calculados pela TC-3D. Exames de RM/ARM devem ser utilizados de forma cautelosa por cirurgiões de ombro no cálculo do glenoid-track.

\section{Suporte Financeiro}

Não houve suporte financeiro de fontes públicas, comerciais, ou sem fins lucrativos.
Conflito de Interesses

Os autores declaram não haver conflito de interesses.

\section{Referências}

1 Momaya AM, Tokish JM. Applying the Glenoid Track Concept in the Management of Patients with Anterior Shoulder Instability. Curr Rev Musculoskelet Med 2017;10(04):463-468

2 França FO, Godinho A, Ribeiro E, Ranzzi A, Bittencourt BL, Barreto BB. Novo método quantitativo para medida da lesão de Hill-Sachs: validação do método radiográfico de Hardy para ressonância magnética/artro-RNM. Rev Bras Ortop 2018;53(05):589-594

3 Di Giacomo G, Itoi E, Burkhart SS. Evolving concept of bipolar bone loss and the Hill-Sachs lesion: from "engaging/non-engaging" lesion to "on-track/off-track" lesion. Arthroscopy 2014;30(01):90-98

4 Gyftopoulos S, Beltran LS, Bookman J, Rokito A. MRI Evaluation of Bipolar Bone Loss Using the On-Track Off-Track Method: A Feasibility Study. AJR Am J Roentgenol 2015;205(04):848-852

5 Metzger PD, Barlow B, Leonardelli D, Peace W, Solomon DJ, Provencher MT. Clinical Application of the "Glenoid Track" Concept for Defining Humeral Head Engagement in Anterior Shoulder Instability: A Preliminary Report. Orthop J Sports Med 2013;1 (02):2325967113496213

6 Bigliani LU, Newton PM, Steinmann SP, Connor PM, Mcllveen SJ. Glenoid rim lesions associated with recurrent anterior dislocation of the shoulder. Am J Sports Med 1998;26(01):41-45

7 Burkhart SS, De Beer JF. Traumatic glenohumeral bone defects and their relationship to failure of arthroscopic Bankart repairs: significance of the inverted-pear glenoid and the humeral engaging Hill-Sachs lesion. Arthroscopy 2000;16(07):677-694

8 Rabinowitz J, Friedman R, Eichinger JK. Management of Glenoid Bone Loss with Anterior Shoulder Instability: Indications and Outcomes. Curr Rev Musculoskelet Med 2017;10(04): 452-462

9 Itoi E, Lee SB, Berglund LJ, Berge LL, An KN. The effect of a glenoid defect on anteroinferior stability of the shoulder after Bankart repair: a cadaveric study. J Bone Joint Surg Am 2000;82(01):35-46

10 Hill HA, Sachs MD. The grooved defect of the humeral head: afrequently unrecognized complication of dislocations of theshoulder joint. Radiology 1940;35:690-700

11 Arciero RA, Parrino A, Bernhardson AS, et al. The effect of a combined glenoid and Hill-Sachs defect on glenohumeral stability: a biomechanical cadaveric study using 3-dimensional modeling of 142 patients. Am J Sports Med 2015;43(06): 1422-1429

12 Kaar SG, Fening SD, Jones MH, Colbrunn RW, Miniaci A. Effect of humeral head defect size on glenohumeral stability: a cadaveric study of simulated Hill-Sachs defects. Am J Sports Med 2010;38 (03):594-599

13 Yamamoto N, Itoi E, Abe H, et al. Contact between the glenoid and the humeral head in abduction, external rotation, and horizontal extension: a new concept of glenoid track. J Shoulder Elbow Surg 2007;16(05):649-656

14 Huijsmans PE, Haen PS, Kidd M, Dhert WJ, van der Hulst VP, Willems WJ. Quantification of a glenoid defect with three-dimensional computed tomography and magnetic resonance imaging: a cadaveric study. J Shoulder Elbow Surg 2007;16(06):803-809

15 Schneider AK, Hoy GA, Ek ET, et al. Interobserver and intraobserver variability of glenoid track measurements. J Shoulder Elbow Surg 2017;26(04):573-579

16 Funakoshi T, Hartzler RU, Stewien E, Burkhart SS. Hill-Sachs Lesion Classification by the Glenoid Track Paradigm in Shoulder Instability: Poor Agreement Between 3-Dimensional Computed Tomographic and Arthroscopic Methods. Arthroscopy 2019;35 (06):1743-1749 